Supporting Information

\title{
Expedient Synthesis of Epigoitrin from L-Ascorbic Acid
}

\author{
Jing-Jing Yang ${ }^{\dagger}$, Jian-Zhong $\mathrm{Wu}^{\dagger}$, Chunhua Qiao* \\ ${ }^{\dagger}$ : These two authors contribute equally to this work $\dagger \dagger$ \\ College of Pharmaceutical Science \\ Soochow University \\ 199 Ren-Ai Road, Suzhou 215123, P. R. China \\ qiaochunhua@suda.edu.cn
}

Experimental detail

$. S 2, S 3$

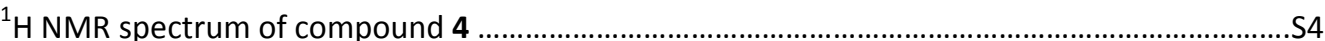

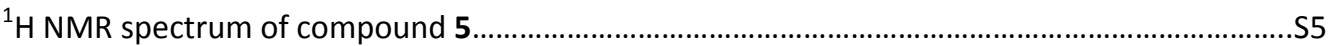

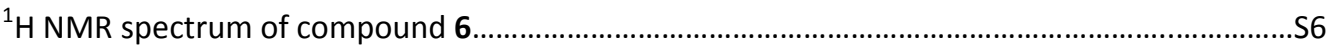

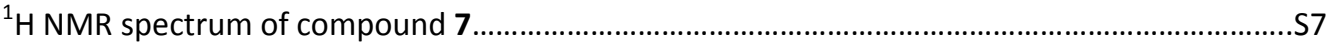

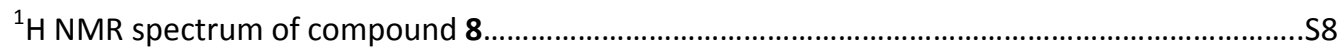

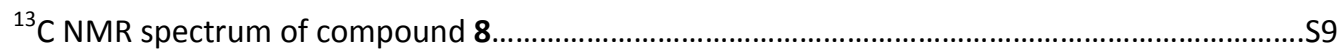

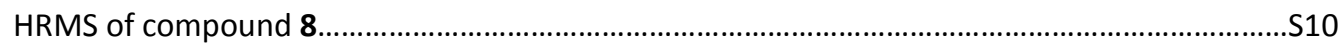

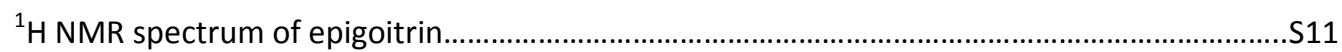

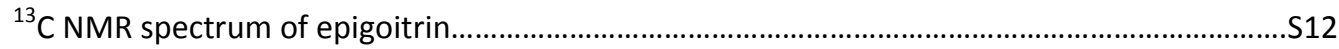

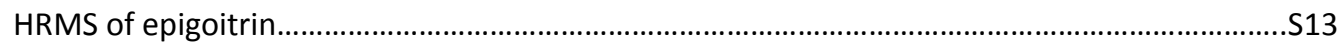


(S)-2,2-Dimethyl-1,3-dioxolane-4-carbaldehyde (4). Compound 4 was prepared from $L$-ascorbic acid according to literature reported method. ${ }^{[15,16]}$ The overall 3 step yield is $50 \%$.

(R)-2,2-Dimethyl-4-vinyl-1,3-dioxolane (5). To an ice cooled solution of potassium tert-butoxide $(0.96 \mathrm{~g}, \quad 8.57 \mathrm{mmol})$ in $10 \mathrm{~mL}$ dry $\mathrm{THF}$ was added methyltriphenylphosphosphonium bromide $(3.06 \mathrm{~g}, 8.57 \mathrm{mmol})$ under nitrogen atmosphere. The solution was stirred for $1.5 \mathrm{~h}$, then compound $4(1.01 \mathrm{~g}, 7.79 \mathrm{mmol})$ in $5 \mathrm{~mL}$ THF was added and the stirring was continued for another $3 \mathrm{~h}$. The reaction was quenched by adding 3 $\mathrm{mL}$ water. The mixture was extracted with ether $(15 \mathrm{~mL} \times 3)$. The combined organic phase was washed with brine, dried over anhydrous $\mathrm{MgSO}_{4}$, concentrated at ambient pressure to remove the ether. The residue was distilled under reduced pressure $(50 \mathrm{~mm})$ at $50^{\circ} \mathrm{C}$ to afford 5 as an oil, $738 \mathrm{mg}(4.21 \mathrm{mmol}, 74 \%)$. The ${ }^{1} \mathrm{H}$ NMR is consistent with literature. ${ }^{[16]}{ }^{1} \mathrm{H}$ NMR (400 MHz, $\left.\mathrm{CDCl}_{3}\right) \delta 5.80(\mathrm{~m}, 1 \mathrm{H}), 5.33(\mathrm{~d}, J=17.1 \mathrm{~Hz}, 1 \mathrm{H}), 5.20(\mathrm{~d}, J=10.3 \mathrm{~Hz}, 1 \mathrm{H})$, $4.48(\mathrm{~m}, 1 \mathrm{H}), 4.08(\mathrm{t}, J=7.1 \mathrm{~Hz}, 1 \mathrm{H}), 3.58$ (t, $J=7.9 \mathrm{~Hz}, 1 \mathrm{H}), 1.41$ (s, 3H), 1.38 (s, 3H).

(R)-But-3-ene-1,2-diol (6). To a stirred solution of 5 (12 mg, $93.63 \mu \mathrm{mol})$ in $1.5 \mathrm{~mL}$ absolute $\mathrm{MeOH}$ was added $p$-toluenesulfonic acid $(17.2 \mathrm{mg}, 100 \mu \mathrm{mol})$ at $0^{\circ} \mathrm{C}$. The reaction was then allowed to warm up to ambient temperature and stirred for another $12 \mathrm{~h}$. It was concentrated in vacuo, the residue was subjected to silica gel flash chromatography $(\mathrm{PE}:$ EtOAc $=2: 1)$ to afford 6 as a colorless oil, $7.9 \mathrm{mg}(89.5 \mu \mathrm{mol}, 96 \%)$. ${ }^{1} \mathrm{H}$ NMR $\left(400 \mathrm{MHz}, \mathrm{CDCl}_{3}\right) \delta 5.82$ (ddd, $J=15.7,10.9,5.0 \mathrm{~Hz}, 1 \mathrm{H}), 5.33(\mathrm{~d}, J=17.3 \mathrm{~Hz}, 1 \mathrm{H}), 5.24-5.15(\mathrm{~d}, J=17.3 \mathrm{~Hz}, 1 \mathrm{H})$, $4.23(\mathrm{~s}, 1 \mathrm{H}), 3.68-3.41(\mathrm{~m}, 3 \mathrm{H}), 3.20(\mathrm{~s}, 1 \mathrm{H})$.

(R)-2-Hydroxylbut-3-enyl 4-methylbenzenesulfonate (7). To a stirred solution of compound 6 (54 mg, $613 \mu \mathrm{mol})$ and anhydrous pyridine $(0.3 \mathrm{~mL}, 3.68 \mathrm{mmol})$ in $2 \mathrm{~mL}$ dry DCM was added tosylchloride $(117 \mathrm{mg}, 613 \mu \mathrm{mol})$. The reaction mixture was stirred for $12 \mathrm{~h}$. The reaction was quenched with $3 \mathrm{~mL}$ saturated $\mathrm{NaHCO}_{3}$ aqueous solution, extracted with DCM $(10 \mathrm{~mL} \times 3)$. The pyridine was removed by washing with sat. $\mathrm{CuSO}_{4}$ aqueous solution $(3 \times 5 \mathrm{~mL})$. The organic phases were dried over anhydrous $\mathrm{Na}_{2} \mathrm{SO}_{4}$ and concentrated in vacuo. The crude product was purified by flash chromatography on silica gel $(\mathrm{PE}:$ EtOAc $=10: 1)$ to give 7 as a white solid, $95 \mathrm{mg}(466 \mu \mathrm{mol}, 76 \%) . \quad[\alpha]_{D}^{25}=+11^{\circ}(\mathrm{c}=1$, acetone $),{ }^{1} \mathrm{H}^{\mathrm{N} M R}{ }^{[16]}$ $\left(400 \mathrm{MHz}, \mathrm{CDCl}_{3}\right) \delta 7.79(\mathrm{~d}, J=8.1 \mathrm{~Hz}, 2 \mathrm{H}), 7.35(\mathrm{~d}, J=7.9 \mathrm{~Hz}, 2 \mathrm{H}), 5.74-5.71(\mathrm{~m}, 1 \mathrm{H})$, 
$5.36(\mathrm{~d}, J=17.2 \mathrm{~Hz}, 1 \mathrm{H}), 5.24(\mathrm{t}, J=8.5 \mathrm{~Hz}, 1 \mathrm{H}), 4.38(\mathrm{~s}, 1 \mathrm{H}), 4.05(\mathrm{dd}, J=10.1,3.4 \mathrm{~Hz}$ $1 \mathrm{H}), 3.90(\mathrm{dd}, J=10.1,7.5 \mathrm{~Hz}, 1 \mathrm{H}), 2.44(\mathrm{~s}, 3 \mathrm{H})$.

(R)-2-(2-Hydroxylbut-3-enyl)isoindoline-1,3-dione (8). A mixture of potassium phthalimide (46 mg, $247.7 \mu \mathrm{mol})$ and compound 7 (30 mg, $123.9 \mu \mathrm{mol})$ was dissolved in 1.0 $\mathrm{mL}$ DMF. The reaction mixture was heated to reflux overnight. The reaction was quenched with $2 \mathrm{~mL}$ water, and the aqueous solution was extracted with diethyl ether $(3 \times 5 \mathrm{~mL})$. The combined organic phase was washed with brine, dried over $\mathrm{Na}_{2} \mathrm{SO}_{4}$ and concentrated in vacuo. The residue was subjected to silica gel flash chromatography $(\mathrm{PE}:$ EtOAc $=10: 1)$ to give compound 8 as yellow solid, $23 \mathrm{mg}(109 \mu \mathrm{mol}, 88 \%) .[\alpha]_{D}^{25}=-2^{\circ}(\mathrm{c}=1 \text {, DCM })^{1} \mathrm{H}$ NMR $\left(300 \mathrm{MHz}, \mathrm{CDCl}_{3}\right): 7.86(\mathrm{~d}, J=6.0 \mathrm{~Hz}, 2 \mathrm{H}), 7.74(\mathrm{~d}, J=6.0 \mathrm{~Hz}, 2 \mathrm{H}), 5.94(\mathrm{ddd}, J=17.2$, 10.3, 7.0 Hz, 1H), $5.40(\mathrm{~d}, J=17.1 \mathrm{~Hz}, 1 \mathrm{H}), 5.22-5.19(\mathrm{~m}, 1 \mathrm{H}), 4.47(\mathrm{~m}, 1 \mathrm{H}), 3.86(\mathrm{~m}$, 2H). ${ }^{13} \mathrm{C}$ NMR $\left(75 \mathrm{MHz}, \mathrm{CDCl}_{3}\right) \delta 169.32,137.82,134.65,132.39,123.96,117.32,71.71$, 44.31. HRMS cacul. for $\left[\mathrm{M}+\mathrm{Na}^{+}\right]: 240.0631$, found[ESI $\left.{ }^{+}\right]: 240.0633$; error $0.92 \mathrm{ppm}$.

(R)-5-vinyloxazolidine-2-thione (Epigoitrin). To a solution of $\mathrm{MeNH}_{2}$ in $\mathrm{EtOH}$ (27-32\% w/w, $0.5 \mathrm{~mL})$ was added compound $8(30 \mathrm{mg}, 0.138 \mathrm{mmol})$. The mixture was stirred at ambient temperature for $12 \mathrm{~h}$ and a large amount of white solid was formed. The precipitation was filtrated and the crude product $\mathbf{9}$ was directly used for the next step reaction. Compound $\mathbf{9}$ was dissolved in $1.0 \mathrm{~mL}$ dry THF, TCDI (thiocarbonyl diimidazole) $(24.6 \mathrm{mg}, 138 \mu \mathrm{mol})$ was added. The reaction mixture was stirred at ambient temperature for $1 \mathrm{~h}$, and was concentrated, the residue was subjected to silica gel flash chromatography $(\mathrm{PE}$ : EtOAc $=8: 1)$ to give epigoitrin as white solid, $12 \mathrm{mg}(93.02 \mu \mathrm{mol}, 70 \%) \cdot[\mathrm{a}]_{\mathrm{D}}^{23.1}=+71^{\circ}\left(\mathrm{c}=1, \mathrm{CHCl}_{3}\right)$, reported $[\alpha]_{D}^{23.1}=+71^{\circ}\left(\mathrm{c}=1, \mathrm{CHCl}_{3}\right) .{ }^{1} \mathrm{H} \mathrm{NMR}\left(300 \mathrm{MHz}, \mathrm{CDCl}_{3}\right) \delta 8.23(\mathrm{br}, 1 \mathrm{H}), 5.94(\mathrm{ddd}, J=17.2$, 10.3, 7.0 Hz, 1H), $5.46(\mathrm{~d}, J=17.1 \mathrm{~Hz}, 1 \mathrm{H}), 5.36(\mathrm{~d}, J=10.4 \mathrm{~Hz}, 1 \mathrm{H}), 5.31-5.28(\mathrm{~m}, 1 \mathrm{H})$, $3.92(\mathrm{t}, J=9.4 \mathrm{~Hz}, 1 \mathrm{H}), 3.53(\mathrm{t}, J=9.4 \mathrm{~Hz}, 1 \mathrm{H}) .{ }^{13} \mathrm{C} \mathrm{NMR}\left(101 \mathrm{MHz}, \mathrm{CDCl}_{3}\right) \delta 189.61$, 132.90, 120.57, 83.35, 49.06. HRMS cacul. for $[\mathrm{M}+\mathrm{H}]^{+}:$130.0321, found[ESI $\left.{ }^{+}\right]: 130.0330$; error $6.97 \mathrm{ppm}$. 
Compound 4. ${ }^{1} \mathrm{H}$ NMR $\left(400 \mathrm{MHz}, \mathrm{CDCl}_{3}\right) \delta 9.72(\mathrm{~d}, J=1.4 \mathrm{~Hz}, 1 \mathrm{H}), 4.39$ (ddd, $J=6.0,4.1,2 \mathrm{~Hz}$, $1 \mathrm{H}), 4.17$ (dd, $J=8.7,4.7 \mathrm{~Hz}, 1 \mathrm{H}), 4.10$ (dd, $J=8.7,4.7 \mathrm{~Hz}, 1 \mathrm{H}), 1.47$ (s, 3H), 1.42 (s, $3 \mathrm{H})$.

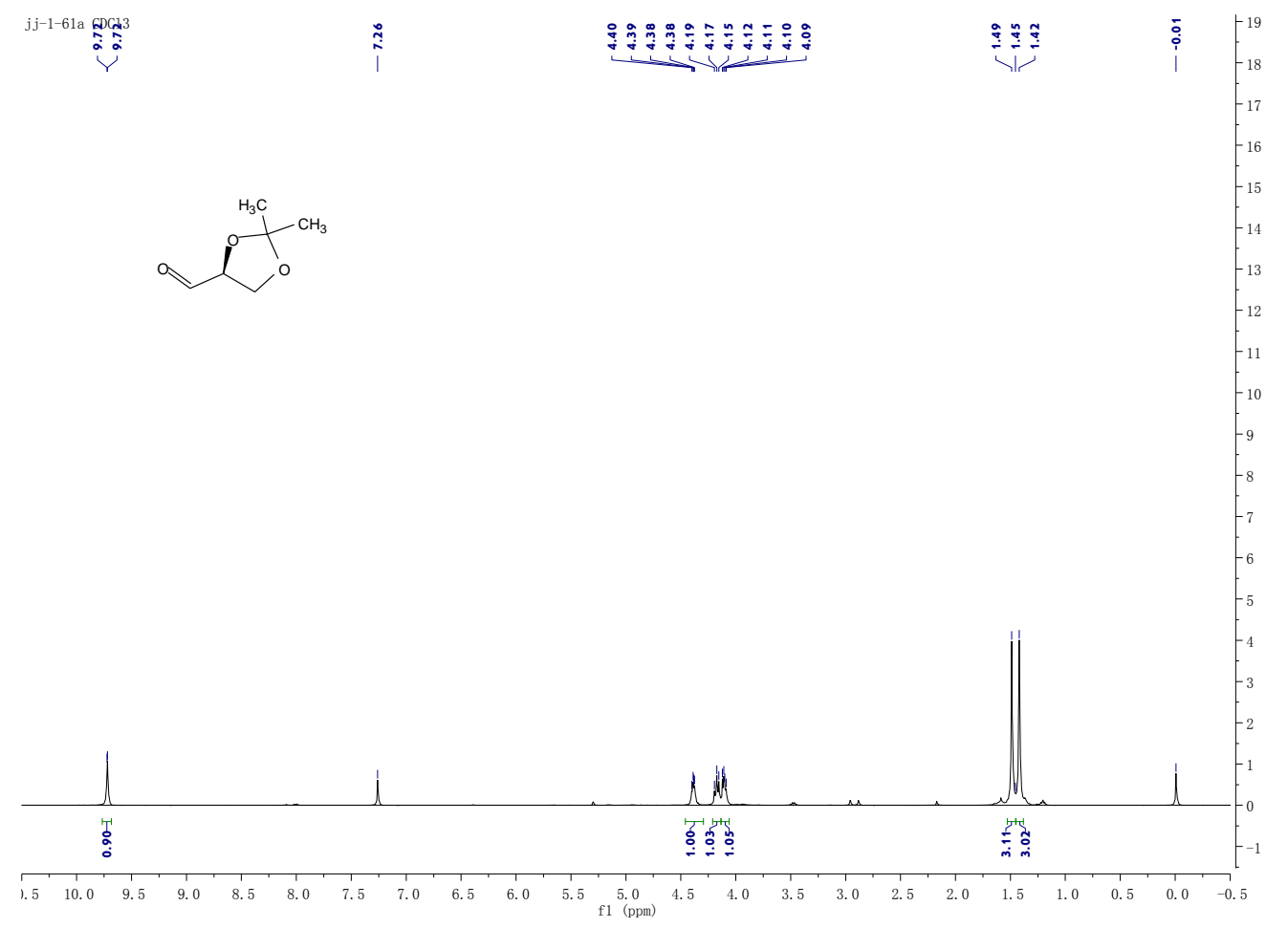


Compound 5. ${ }^{1} \mathrm{H}$ NMR (400 MHz, $\left.\mathrm{CDCl}_{3}\right) \delta 5.80(\mathrm{~m}, 1 \mathrm{H}), 5.33(\mathrm{~d}, J=17.1 \mathrm{~Hz}, 1 \mathrm{H}), 5.20(\mathrm{~d}, J=$ $10.3 \mathrm{~Hz}, 1 \mathrm{H}), 4.48(\mathrm{~m}, 1 \mathrm{H}), 4.08(\mathrm{t}, J=7.1 \mathrm{~Hz}, 1 \mathrm{H}), 3.58(\mathrm{t}, J=7.9 \mathrm{~Hz}, 1 \mathrm{H}), 1.41(\mathrm{~s}, 3 \mathrm{H}), 1.38(\mathrm{~s}, 3 \mathrm{H})$.

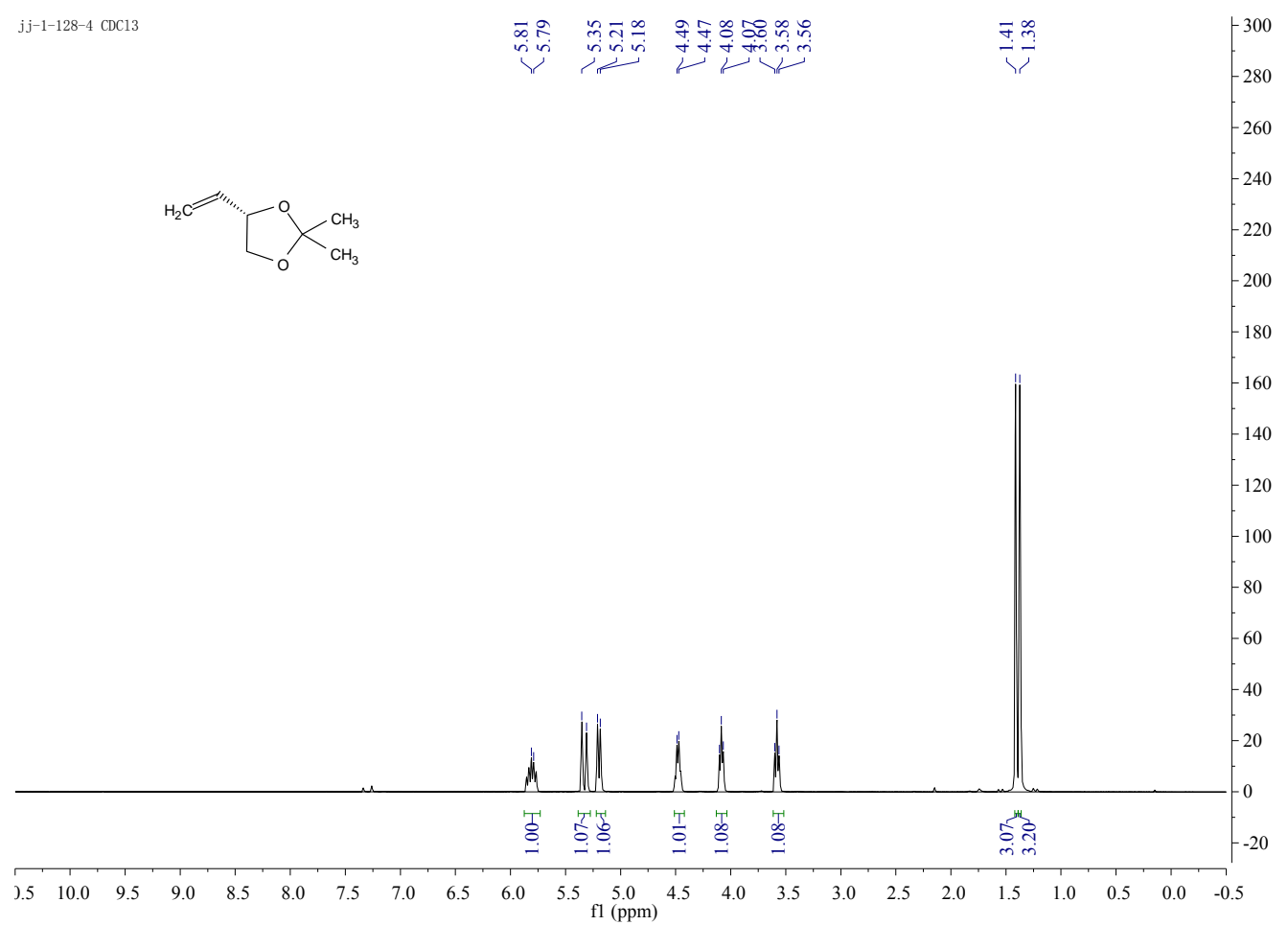


Compound 6. ${ }^{1} \mathrm{H}$ NMR $\left(400 \mathrm{MHz}, \mathrm{CDCl}_{3}\right) \delta 5.82(\mathrm{ddd}, J=15.7,10.9,5.0 \mathrm{~Hz}, 1 \mathrm{H}), 5.33(\mathrm{~d}, J=17.3$

$\mathrm{Hz}, 1 \mathrm{H}), 5.21-5.18(\mathrm{~d}, J=17.3 \mathrm{~Hz}, 1 \mathrm{H}), 4.23(\mathrm{~s}, 1 \mathrm{H}), 3.65-3.44$ (m, 3H), $3.20(\mathrm{~s}, 1 \mathrm{H})$.

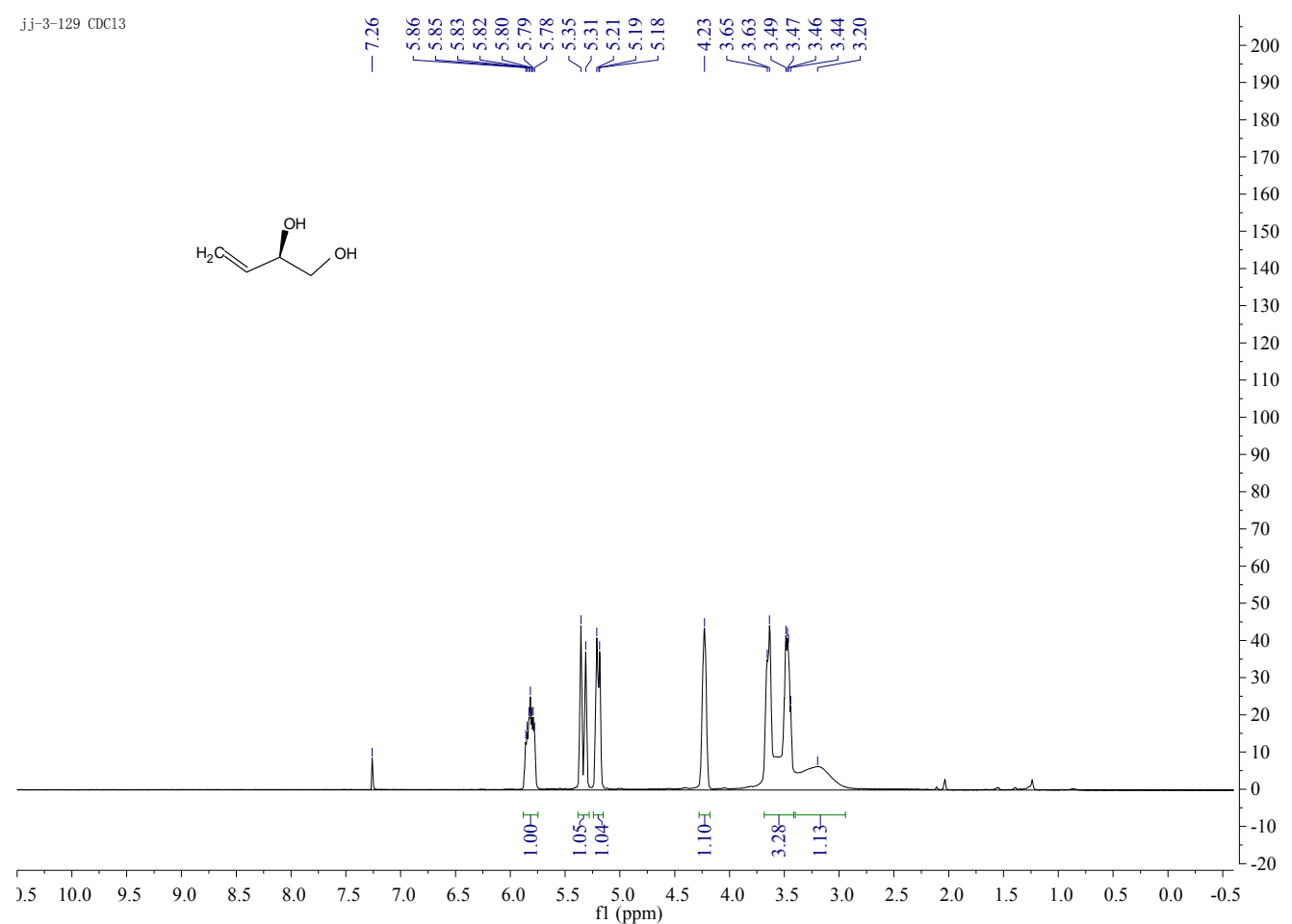


Compound 7. ${ }^{1} \mathrm{H} \mathrm{NMR}^{[16]}\left(400 \mathrm{MHz}, \mathrm{CDCl}_{3}\right) \delta 7.79(\mathrm{~d}, J=8.1 \mathrm{~Hz}, 2 \mathrm{H}), 7.35(\mathrm{~d}, J=7.9 \mathrm{~Hz}, 2 \mathrm{H})$, $5.74-5.71(\mathrm{~m}, 1 \mathrm{H}), 5.36(\mathrm{~d}, J=17.2 \mathrm{~Hz}, 1 \mathrm{H}), 5.24(\mathrm{t}, J=8.5 \mathrm{~Hz}, 1 \mathrm{H}), 4.38(\mathrm{~s}, 1 \mathrm{H}), 4.05(\mathrm{dd}, J=10.1$, $3.4 \mathrm{~Hz}, 1 \mathrm{H}), 3.90$ (dd, $J=10.1,7.5 \mathrm{~Hz}, 1 \mathrm{H}), 2.44$ (s, 3H).

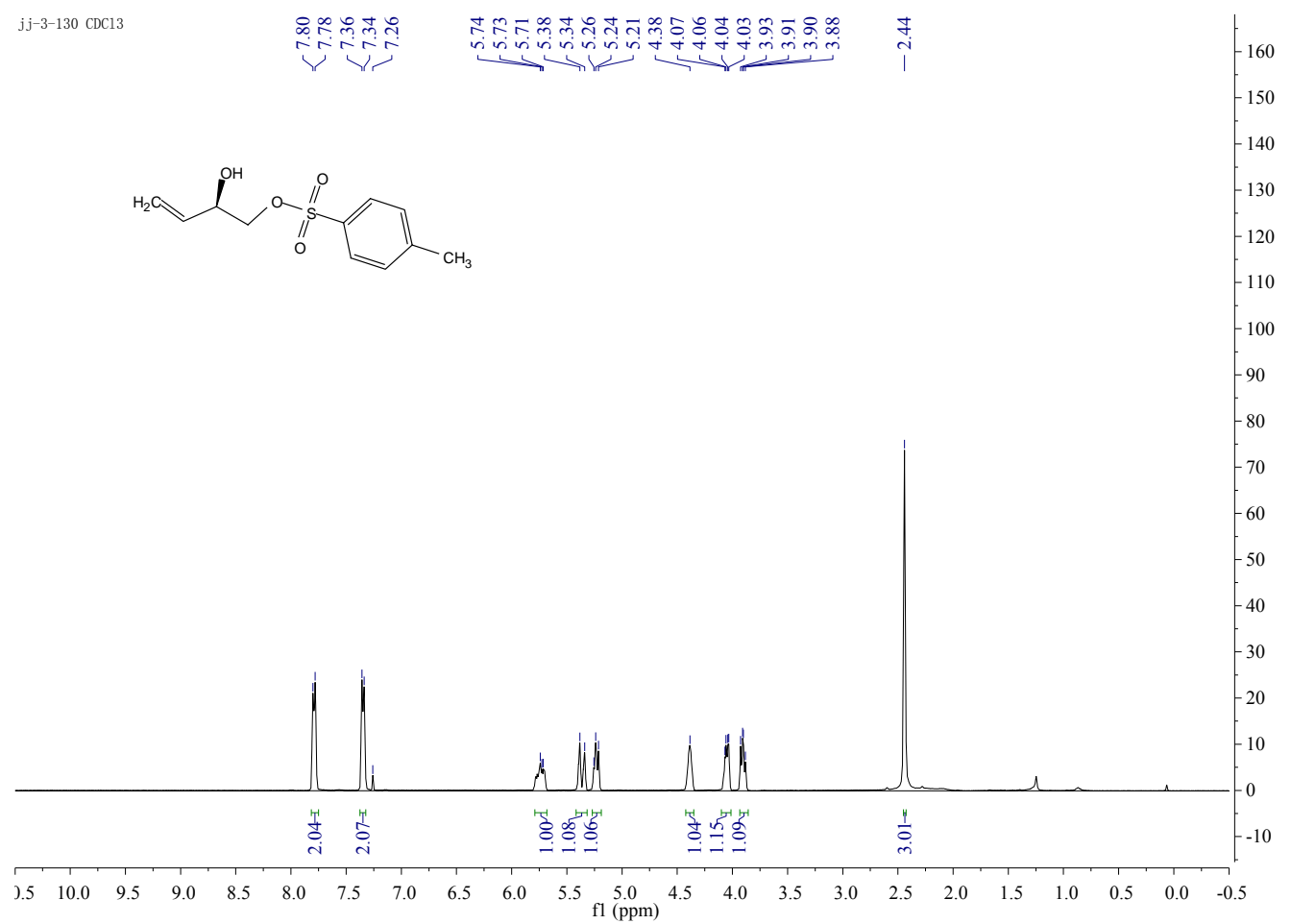


Compound 8. ${ }^{1} \mathrm{H}$ NMR (300 MHz, $\left.\mathrm{CDCl}_{3}\right): 7.86(\mathrm{~d}, J=6.0 \mathrm{~Hz}, 2 \mathrm{H}), 7.74(\mathrm{~d}, J=6.0 \mathrm{~Hz}, 2 \mathrm{H}), 5.94$ (ddd, $J=17.2,10.3,7.0 \mathrm{~Hz}, 1 \mathrm{H}), 5.40(\mathrm{~d}, J=17.1 \mathrm{~Hz}, 1 \mathrm{H}), 5.22-5.19(\mathrm{~m}, 1 \mathrm{H}), 4.47$ (m, 1H), $3.86(\mathrm{~m}$, $2 \mathrm{H})$.

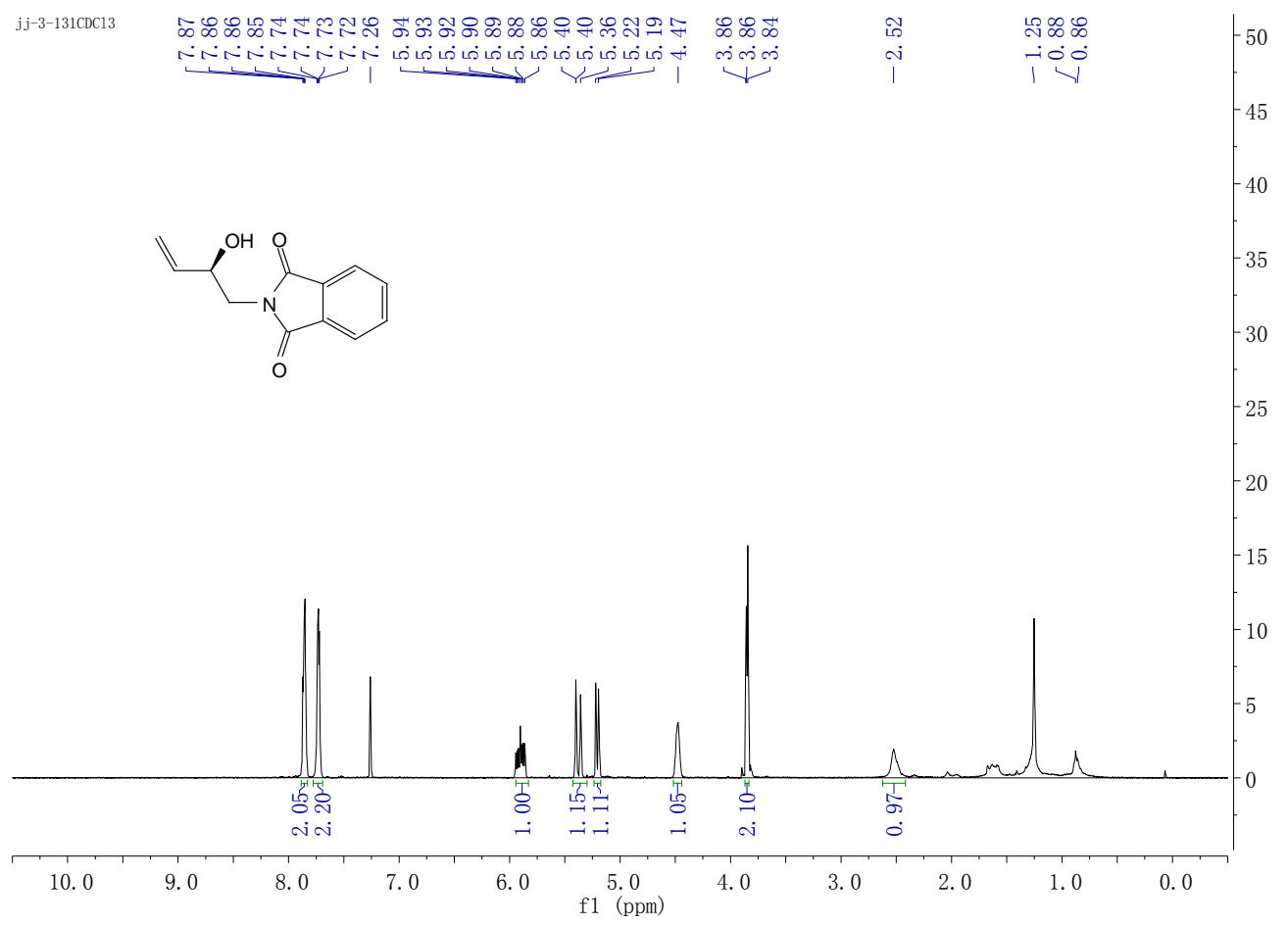


${ }^{13} \mathrm{C}$ NMR spectrum of compound $8{ }^{13} \mathrm{C}$ NMR $\left(75 \mathrm{MHz}, \mathrm{CDCl}_{3}\right) \delta 169.32,137.82,134.65,132.39$, 123.96, 117.32, 71.71, 44.31 .

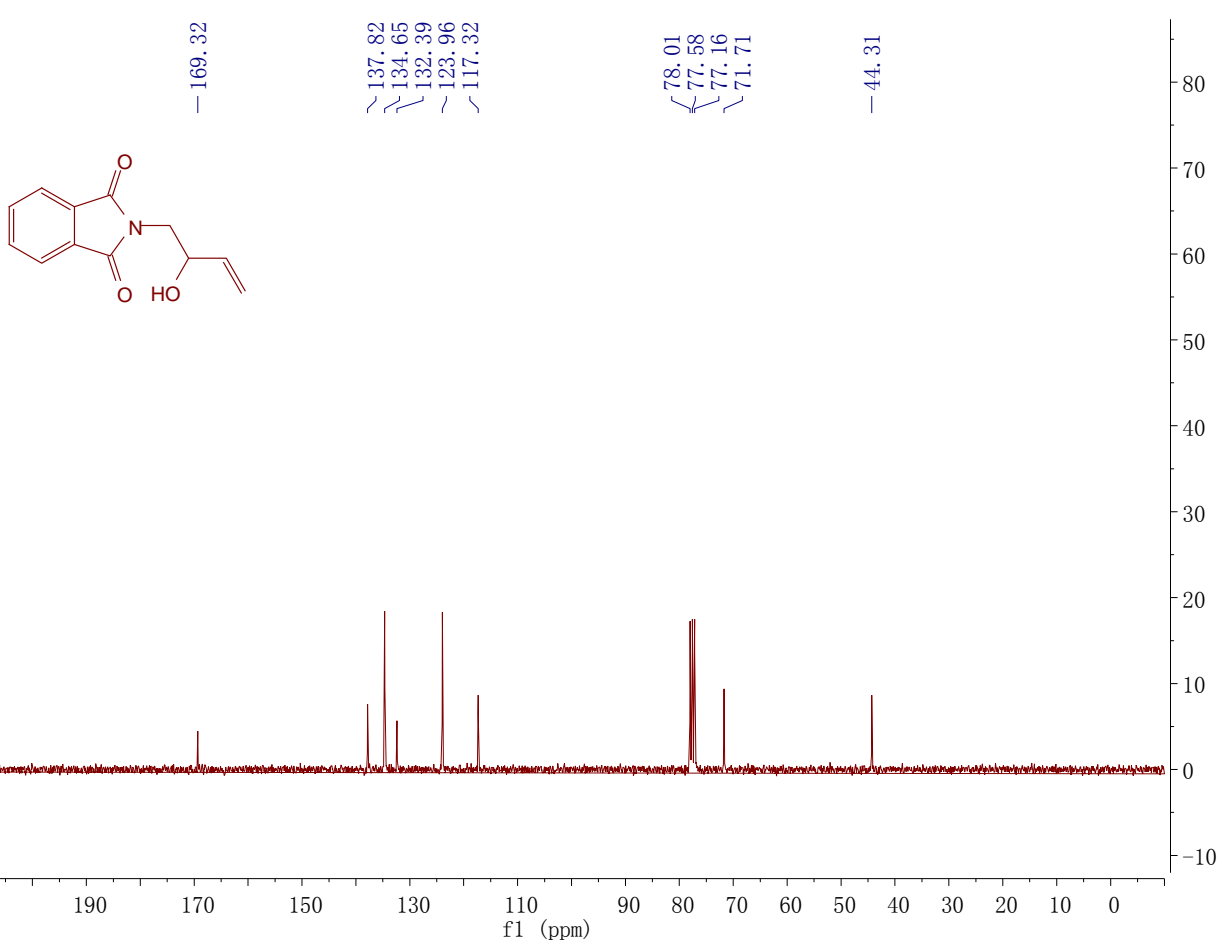


HRMS of compound 8

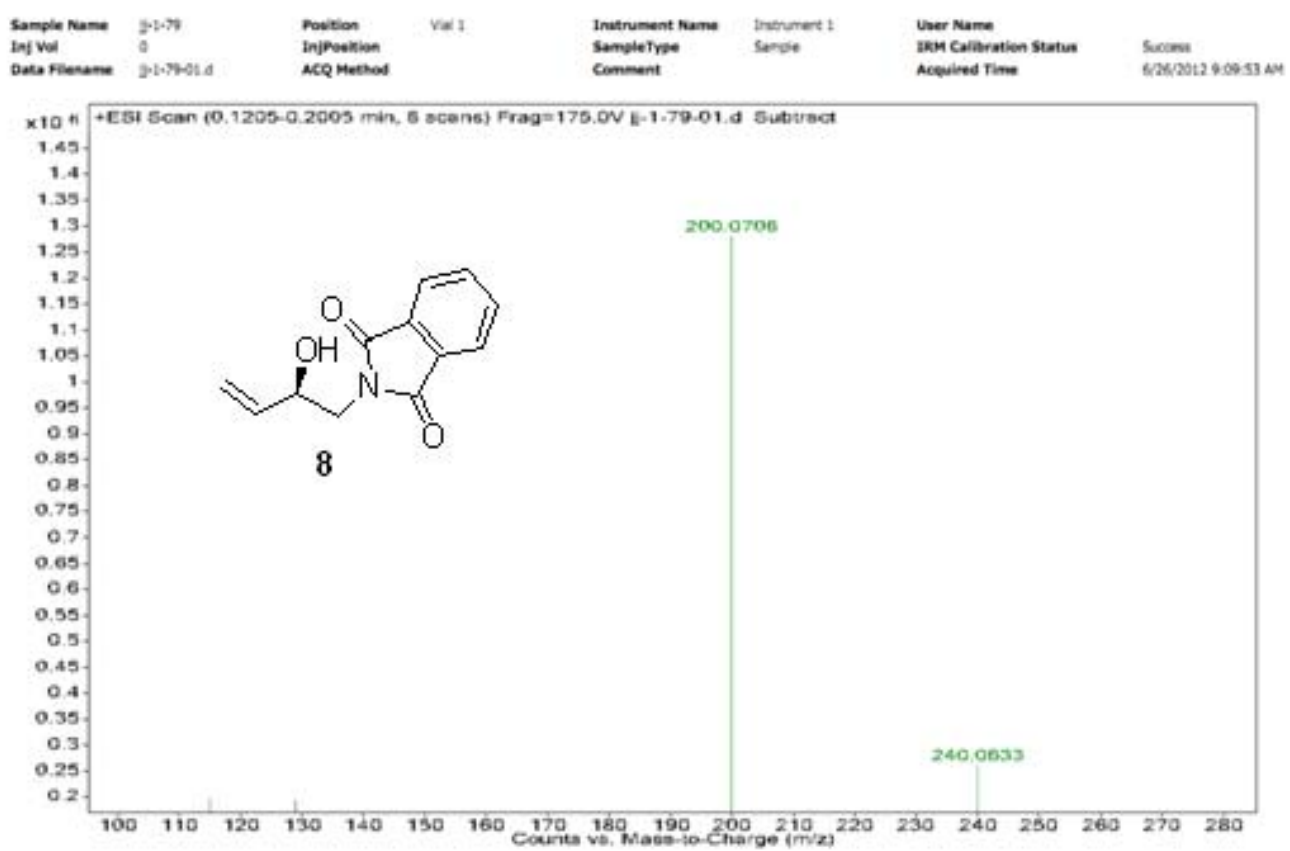


${ }^{1} \mathrm{H}$ NMR spectrum of epigoitrin $\quad{ }^{1} \mathrm{H}$ NMR $\left(300 \mathrm{MHz}, \mathrm{CDCl}_{3}\right) \delta 8.23(\mathrm{br}, 1 \mathrm{H}), 5.94$ (ddd, $J=17.2$, $10.3,7.0 \mathrm{~Hz}, 1 \mathrm{H}), 5.46(\mathrm{~d}, J=17.1 \mathrm{~Hz}, 1 \mathrm{H}), 5.36(\mathrm{~d}, J=10.4 \mathrm{~Hz}, 1 \mathrm{H}), 5.31-5.28(\mathrm{~m}, 1 \mathrm{H}), 3.92(\mathrm{t}, J=$ $9.4 \mathrm{~Hz}, 1 \mathrm{H}), 3.53(\mathrm{t}, J=9.4 \mathrm{~Hz}, 1 \mathrm{H})$.

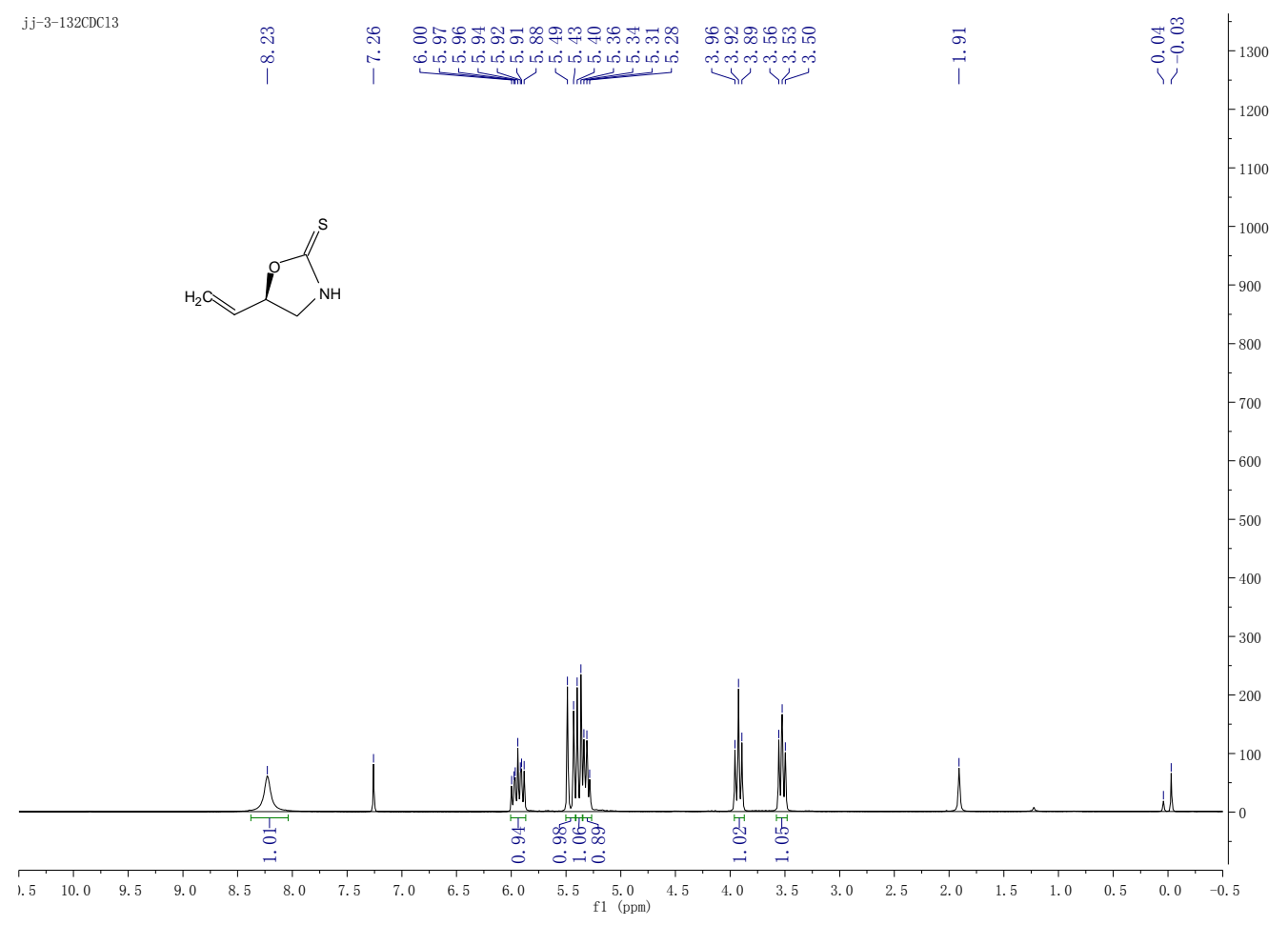




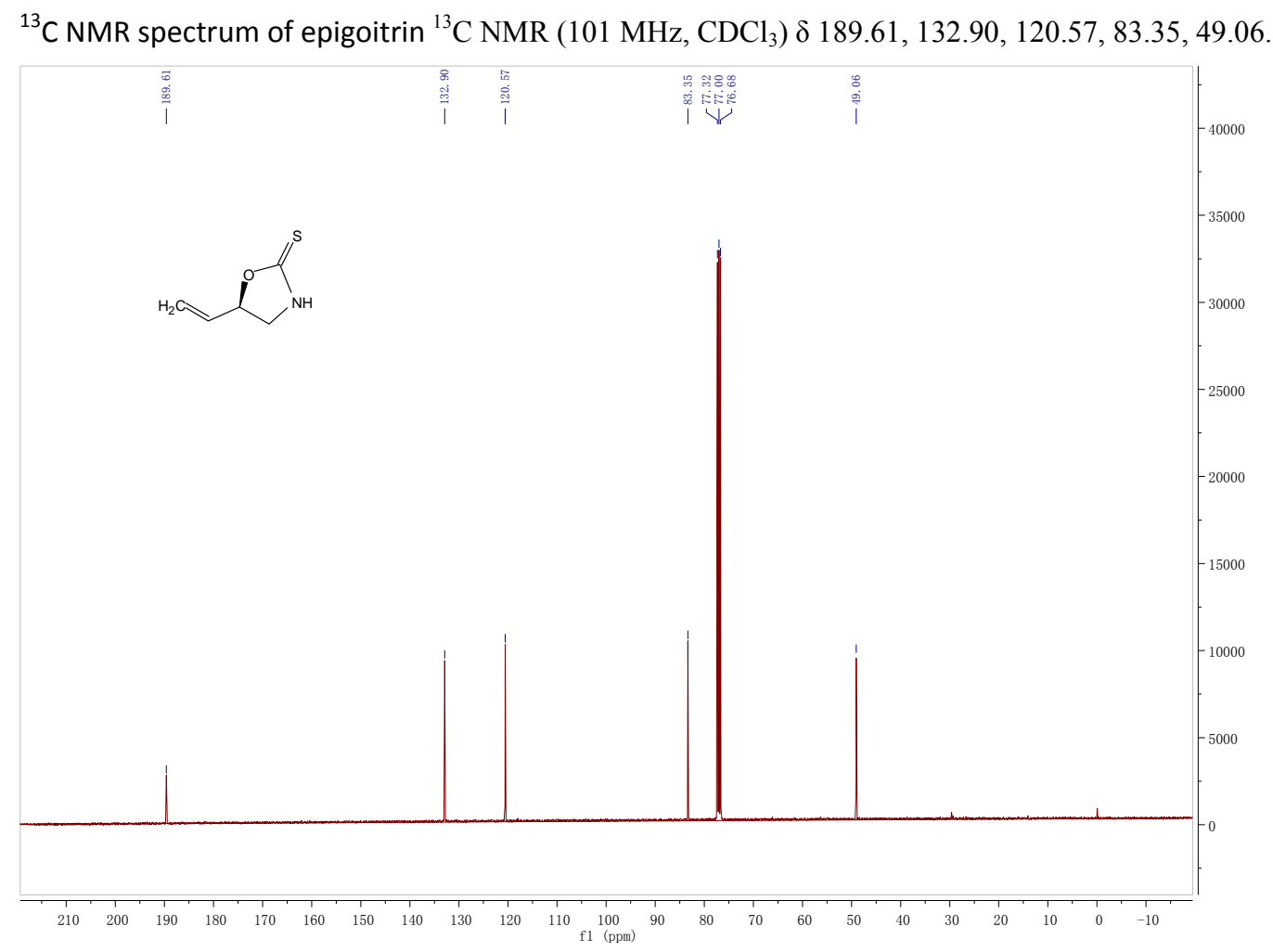




\section{HRMS of epigoitrin}

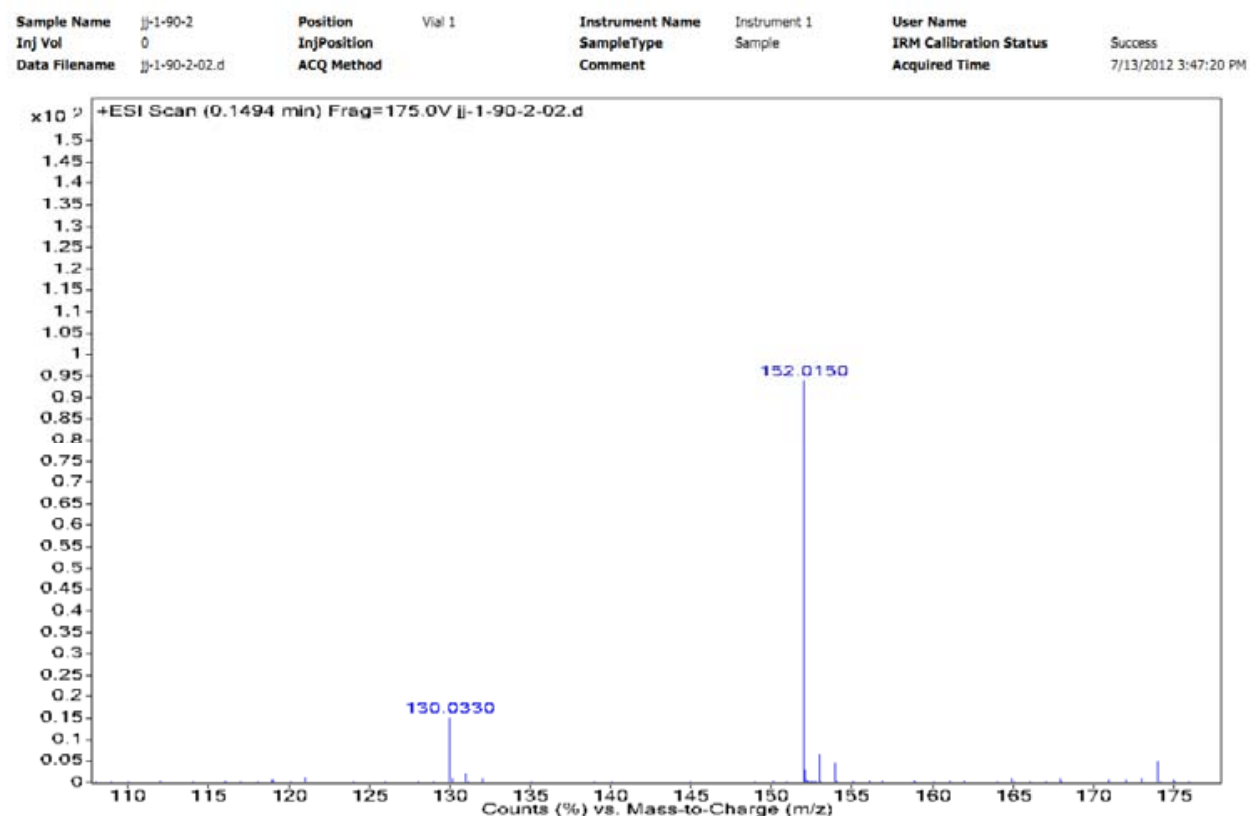

